\title{
A NOTE ON THE FOURTH POWER MOMENT OF THE RIEMANN ZETA-FUNCTION
}

\author{
J.B. CONREY
}

ABstract. We give explicit formulae for all of the terms in the asymptotic expansion of the mean fourth power of the Riemann zeta-function on the critical line.

Heath-Brown has evaluated the 4th power moment of the Riemann zeta-function on the critical line via the formula

$$
I_{2}(T)=\int_{0}^{T}|\zeta(1 / 2+i t)|^{4} d t=T P_{4}\left(\log \frac{T}{2 \pi}\right)+O\left(T^{7 / 8+\epsilon}\right)
$$

where $P_{4}$ is a 4 th degree polynomial. Here we give an explicit formula for $P_{4}$. In particular, we show that the coefficients of $P_{4}$ are in the field

$$
Q\left(\gamma_{0}, \gamma_{1}, \gamma_{2}, \gamma_{3}, \zeta(2), \zeta^{\prime}(2), \zeta^{\prime \prime}(2)\right)
$$

where the $\gamma$ 's are the coefficients in the Laurent expansion

$$
\zeta(s)=\frac{1}{s-1}+\gamma_{0}+\gamma_{1}(s-1)+\ldots
$$

of $\zeta(s)$ at $s=1$.

Theorem. We have

$$
P_{4}(x)=g_{0}(x)+g_{1}(x)
$$

where

$$
g_{0}(x)=\operatorname{Res}_{s=0} \frac{2 x^{s} \zeta(s+1)^{4}}{s(s+1) \zeta(2 s+2)}
$$

and

$$
g_{1}(x)=\left.\left(\frac{d}{d s}\right)^{2} \frac{\left(x e^{2 \gamma_{0}}\right)^{s}\left(\frac{1}{2} \zeta(s+1)^{2}-\frac{1}{s} \zeta(2 s+1)-\zeta(2 s+2)\right)}{(s+1) \zeta(s+2)}\right|_{s=0} .
$$

Ivic has also written down formulae for the coefficients of $P_{4}$.

Proof. We refer to Heath-Brown's paper. There he shows that $P_{4}$ is naturally expressed as a sum of $g_{0}+g_{1}$. The first term $g_{0}$ arises from the diagonal terms

Research supported in part by a grant from the NSF. 
and is exactly as above. The other piece arises from the off diagonal terms and it is the explicit calculation of this part that is the purpose of this paper. Following Heath-Brown, we write

$$
\sum_{n \leq x} d(n) d(n+r)=m(x, r)+E(x, r)
$$

where $m(x, r) / x$ is a polynomial of degree 2 in $x$ with coefficients depending on $r$. In fact,

$$
m(x, r)=\operatorname{Res}_{s=1} D(s, r) \frac{x^{s}}{s}
$$

with

$$
D(r, s)=\sum_{n=1}^{\infty} \frac{d(n) d(n+r)}{n^{s}}
$$

Then

$$
g_{1}\left(\frac{T}{2 \pi}\right)=2 \sum_{r=1}^{\infty} \int_{0}^{T /(2 \pi)} m^{\prime}(x, r) \sin (T r / x) d x .
$$

Lemma. With the above notation,

$$
m(x, r)=\sum_{s=1}^{\infty} \frac{\mu(s)}{s^{2}} \sum_{d \mid r}\left(\left(\log \frac{x}{d^{2} s^{2}}+2 \gamma_{0}-1\right)^{2}+1\right)
$$

and

$$
m^{\prime}(x, r)=\sum_{s=1}^{\infty} \frac{\mu(s)}{s^{2}} \sum_{d \mid r}\left(\log \frac{x}{d^{2} s^{2}}+2 \gamma_{0}\right)^{2}
$$

Proof. According to Heath-Brown, $m(x, r)$ may be calculated by considering the main terms in

$$
2 \sum_{q \leq x^{1 / 2}} R(x, q, r)-\sum_{q \leq x^{1 / 2}} R\left(q x^{1 / 2}, q, r\right)
$$

where

$$
R(x, q, r)=\frac{x}{q^{2}} \sum_{d \mid(q, r)} \sum_{\delta \mid q / d} d \delta \mu(q / d \delta)\left(\log \frac{x \delta^{2}}{q^{2}}+2 \gamma_{0}-1\right) .
$$

Writing $q=s d \delta$ we have

$$
\sum_{q \leq x^{1 / 2}} R(x, q, r)=x \sum_{d \mid r} \frac{1}{d} \sum_{s \leq x^{1 / 2} / d} \frac{\mu(s)}{s^{2}}\left(\log \frac{x}{d^{2} s^{2}}+2 \gamma_{0}-1\right) \sum_{\delta \leq x^{1 / 2} / s d} \frac{1}{\delta}
$$

Using

$$
\sum_{\delta \leq y} \frac{1}{\delta}=\log y+\gamma_{0}+O(1 / y)
$$


(and we note

$$
\sum_{\delta \leq y} \frac{\log \delta}{\delta}=\frac{1}{2} \log ^{2} y-\gamma_{1}+O(\log y / y),
$$

for future reference) we find that the above sum is

$$
\begin{gathered}
=\frac{x}{2} \sum_{d \mid r} \frac{1}{d} \sum_{s \leq x^{1 / 2} / d} \frac{\mu(s)}{s^{2}}\left(\log \frac{x}{d^{2} s^{2}}+2 \gamma_{0}-1\right)\left(\log \frac{x}{d^{2} s^{2}}+2 \gamma_{0}\right) \\
+O\left(x^{1 / 2} \sum_{d \mid r} \sum_{s \leq x^{1 / 2}} \frac{1}{s}\right) .
\end{gathered}
$$

Extending the sum over $s$ to $\infty$ the above is

$$
=\sum_{s=1}^{\infty} \frac{\mu(s)}{s^{2}} \sum_{d \mid r}\left(\log \frac{x}{d^{2} s^{2}}+2 \gamma_{0}-1\right)\left(\log \frac{x}{d^{2} s^{2}}+2 \gamma_{0}\right)+O\left(x^{1 / 2} \log x d(r)\right) .
$$

Similarly, using

$$
\sum_{\delta \leq y} 1=y+O(1)
$$

and

$$
\sum_{\delta \leq y} \log \delta=y \log y-y+O(\log y)
$$

we find that the main part of

$$
\sum_{q \leq x^{1 / 2}} R\left(x^{1 / 2} q, q, r\right)
$$

is given by

$$
\sum_{s=1}^{\infty} \frac{\mu(s)}{s^{2}} \sum_{d \mid r}\left(\log \frac{x}{d^{2} s^{2}}+2 \gamma_{0}-2\right)
$$

Putting these two calculations together, we obtain the formula for $m(x, r)$. Differentiating with respect to $x$ gives the rest of the lemma.

Now we compute

$$
g_{1}=2 \sum_{r=1}^{\infty} \int_{0}^{T /(2 \pi)} m^{\prime}(x, r) \sin (\operatorname{Tr} / x) d x
$$

To begin with, we change variables, letting $y=\operatorname{Tr} / x$. Then, substituting the expression for $m^{\prime}(x, r)$ from the lemma gives

$$
g_{1}=2 \sum_{r=1}^{\infty} \int_{2 \pi r}^{\infty} \sum_{s=1}^{\infty} \frac{\mu(s)}{s^{2}} \sum_{d \mid r} \frac{1}{d}\left(\log \frac{T r}{s^{2} d^{2} y}+2 \gamma_{0}-1\right)^{2} \frac{\sin y}{y^{2}} d y
$$


We bring the sum over $s$ to the front, and interchange the summation over $r$ and the integration, and replace $d$ by $n$ and write $r=m n$. Then we have

$$
g_{1}=2 \sum_{s=1}^{\infty} \frac{\mu(s)}{s^{2}} \int_{2 \pi}^{\infty} \sum_{m n \leq y / 2 \pi} \frac{1}{n}\left(\log \frac{T m}{s^{2} n y}+2 \gamma_{0}\right)^{2} \frac{\sin y}{y^{2}} d y .
$$

Next, we interchange the sum over $m$ and the integration giving

$$
g_{1}=2 \sum_{s=1}^{\infty} \frac{\mu(s)}{s^{2}} \sum_{m=1}^{\infty} \int_{2 \pi m}^{\infty} \sum_{n \leq y / 2 \pi m} \frac{1}{n}\left(\log \frac{T m}{s^{2} n y}+2 \gamma_{0}\right)^{2} \frac{\sin y}{y^{2}} d y
$$

A change of variables in the integration, $u=y / 2 \pi m$ gives

$$
g_{1}=2 \sum_{s=1}^{\infty} \frac{\mu(s)}{s^{2}} \sum_{m=1}^{\infty} \int_{1}^{\infty} \sum_{n \leq u} \frac{1}{n}\left(\log \frac{T / 2 \pi}{s^{2} u n}+2 \gamma_{0}\right)^{2} \frac{\sin 2 \pi u m}{2 \pi u m} \frac{d u}{u}
$$

Now,

$$
-\sum_{m=1}^{\infty} \frac{\sin 2 \pi u m}{\pi m}=u-[u]-1 / 2=((u)) .
$$

Thus, bringing the sum over $m$ to the inside, we get

$$
g_{1}=-\sum_{s=1}^{\infty} \frac{\mu(s)}{s^{2}} \int_{1}^{\infty} \sum_{n \leq u} \frac{1}{n}\left(\log \frac{T / 2 \pi}{s^{2} u n}+2 \gamma_{0}\right)^{2}((u)) \frac{d u}{u^{2}} .
$$

Interchanging the sum over $n$ and the integration, and changing $s$ into $m$ leads to

$$
g_{1}=-\sum_{m=1}^{\infty} \frac{\mu(m)}{m^{2}} \sum_{n=1}^{\infty} \int_{n}^{\infty} \frac{1}{n}\left(\log \frac{T / 2 \pi}{m^{2} u n}+2 \gamma_{0}\right)^{2}((u)) \frac{d u}{u^{2}} .
$$

Now we express the logs via differentiation:

$$
g_{1}=-\left(\frac{d}{d s}\right)^{2}\left(\left.\sum_{m=1}^{\infty} \frac{\mu(m)}{m^{2+s}}\left(\frac{T e^{2 \gamma_{0}}}{2 \pi}\right)^{s} \sum_{n=1}^{\infty} \frac{1}{n^{1+s}} \int_{n}^{\infty}((u)) \frac{d u}{u^{2+s}}\right|_{s=0}\right.
$$

It is well known that

$$
-\int_{n}^{\infty} \frac{((u))}{u^{s+2}} d u=\frac{1}{s+1}\left(\zeta(s+1)-\frac{1}{s n^{s}}-\sum_{m=1}^{n} \frac{1}{m^{s+1}}-\frac{1}{2 n^{s+1}}\right) .
$$

Thus,

$$
\begin{aligned}
g_{1}=\lim _{N \rightarrow \infty}-\left(\frac{d}{d s}\right)^{2} & \left(\left(\frac{T e^{2 \gamma_{0}}}{2 \pi}\right)^{s} \frac{1}{(s+1) \zeta(s+2)}\right. \\
& \left.\sum_{n=1}^{N} \frac{1}{n^{1+s}}\left(\zeta(s+1)-\frac{1}{s n^{s}}-\sum_{m=1}^{n} \frac{1}{m^{s+1}}-\frac{1}{2 n^{s+1}}\right)\right|_{s=0} .
\end{aligned}
$$


Now

$$
\sum_{n=1}^{N} \frac{1}{n^{s+1}} \sum_{m=1}^{n} \frac{1}{m^{s+1}}=\sum_{m=1}^{N} \frac{1}{m^{s+1}} \sum_{n=m}^{N} \frac{1}{n^{s+1}}
$$

so that

$$
2 \sum_{n=1}^{N} \frac{1}{n^{s+1}} \sum_{m=1}^{n} \frac{1}{m^{s+1}}=\left(\sum_{n=1}^{N} \frac{1}{n^{s+1}}\right)^{2}+\sum_{n=1}^{N} \frac{1}{n^{2 s+2}} .
$$

Thus, the sum over $n$ in the above expression is

$$
=\zeta(s+1) \sum_{n=1}^{n} \frac{1}{n^{s+1}}-\frac{1}{s} \sum_{n=1}^{N} \frac{1}{n^{2 s+1}}-\frac{1}{2}\left(\sum_{n=1}^{N} \frac{1}{n^{s+1}}\right)^{2}-\sum_{n=1}^{N} \frac{1}{n^{2 s+2}} .
$$

If we assume that $s>0$, we can let $N \rightarrow \infty$ here, getting

$$
\frac{1}{2} \zeta(s+1)^{2}-\frac{1}{s} \zeta(2 s+1)-\zeta(2 s+2)
$$

Inserting this into our prior expresion for $g 1$, we obtain the Theorem.

\section{REFERENCES}

[H-B] D.R. Heath-Brown, The fourth power moment of the Riemann zeta function, J. London Math. Soc (3) 38 (1979), 385-422.

Department of Mathematics, Oklahoma State University, Stillwater, OK 74078 